\title{
SPACE PREEMPTION, SIZE-DEPENDENT COMPETITION, AND THE COEXISTENCE OF CLONAL GROWTH FORMS
}

\author{
Sean R. CONnOlly ${ }^{1,3}$ and Soyoka Muko ${ }^{2}$ \\ ${ }^{1}$ Centre for Coral Reef Biodiversity, Department of Marine Biology, James Cook University, Townsville, \\ Queensland 4811, Australia \\ ${ }^{2}$ Department of Biology, Faculty of Sciences, Kyushu University, Fukuoka 812-8581, Japan
}

\begin{abstract}
Interspecific interactions that produce nontransitive competitive networks have been proposed to promote diversity in a broad range of systems, including coral reefs. In this paper, we model the effect of size-dependent shifts in competitive ability on the coexistence of canopy-forming and understory coral species, and we predict how these shifts influence patterns of community structure along large-scale gradients in disturbance and recruitment limitation. We consider three models, representing a gradient from purely hierarchical competition in which the canopy-former is dominant, to competition involving standoffs and reversals between the understory species and juvenile canopy-formers. Analysis of these models leads to two key conclusions. First, as competition becomes less transitive, coexistence may be promoted or inhibited, depending upon the extent to which the canopy-former can sustain itself by clonal propagation. Specifically, when clonal growth alone is adequate to sustain the canopy-former, increasing nontransitivity promotes coexistence. When it is not, nontransitivity inhibits coexistence. Secondly, size-dependent nontransitivity dramatically changes how gradients in disturbance and recruitment affect species coexistence. In contrast to hierarchical interactions, standoffs and reversals do not show an "intermediate recruitment" phenomenon, in which coexistence is facilitated at intermediate levels of recruitment. Moreover, under hierarchical competition, the dominant always benefits more than the subordinate as recruitment is increasingly facilitated. Under standoffs and reversals, however, increasing recruitment often favors the canopy-former at some levels of disturbance, but the understory species at other levels of disturbance. These results differ markedly from previous models of stage-dependent competition, suggesting that promotion of coexistence by ontogenetic shifts in competitive ability depends upon the mechanisms by which competition occurs in particular ecological contexts. The results also indicate that the effects of gradients in disturbance and recruitment on community structure depend fundamentally on how species compete for space.
\end{abstract}

Key words: benthic competition; community dynamics; competition theory; coral assemblages; coral reefs; intermediate-disturbance hypothesis; intermediate-recruitment hypothesis; nontransitive competition; ontogenetic shifts in competitive ability; recruitment limitation; species coexistence.

\section{INTRODUCTION}

Understanding how species diversity is maintained when many species compete for one or a few common limiting resources is a perennial issue in ecology (Hutchinson 1961, Connell 1978, Hubbell 2001). Much theoretical and experimental work in community ecology has focused on this topic, particularly for sessile organisms. Because the mobility of such species is limited to a specific dispersal phase, access to space-and the resources that come with it (e.g., light, suspended particles)_can be limiting to individuals even when those resources are available elsewhere in the habitat. As a result, models of competition for space have been among the most extensively explored in community ecology (Yu and Wilson 2001).

Manuscript received 10 June 2002; revised 6 January 2003; accepted 30 January 2003; final version received 4 March 2003 Corresponding Editor: M. A. Hixon.

${ }^{3}$ E-mail: sean.connolly@jcu.edu.au
Mathematical theory for assemblages of space-limited, benthic marine animals has tended to adopt one of two characterizations of competition: symmetric, preemptive competition or strictly hierarchical competition (see Sebens [1982] for an exception). Under preemptive competition, once adults gain access to space they cannot be competitively displaced, so all species' larvae are restricted to settling in vacant patches of habitat (Chesson and Warner 1981, Iwasa and Roughgarden 1986, Muko and Iwasa 2000). By contrast, under hierarchical competition the individuals of a dominant species always displace individuals of a subordinate species (Hastings 1980, Stone 1995, Connolly and Roughgarden 1998).

Some benthic assemblages appear well characterized by hierarchical models (e.g., intertidal barnacles and mussels, Connell 1961, Paine 1966; but see Dungan 1985); some by symmetric, preemptive models (e.g., coral-dwelling reef fish, Sale 1977; but see Munday et al. 2001); and others by a combination of hierarchical 
overgrowth and symmetric preemption (e.g., many sessile clonal invertebrates, Karlson 1980, 1983, Sebens 1986). However, more complex competitive relationships occur as well. For instance, communities may exhibit "intransitive loops" in which species A consistently outcompetes species B, B consistently outcompetes species C, but species $\mathrm{C}$ outcompetes species A (Buss and Jackson 1979). In addition, the outcome of competition between two individuals or colonies may depend on factors such as angle of attack, relative size, or physiological condition (Connell 1976, Jackson 1979, Buss 1980). Both of these kinds of more complex relationships produce nontransitive competitive networks, in which there is no clear hierarchy of competitors (e.g., Jackson 1979, Keddy and Shipley 1989). Evidence for such relationships is particularly strong for coral reefs (Buss and Jackson 1979, Jackson 1979, Chornesky 1989, Tanner 1992, Tanner et al. 1994, 1996), and this network-like complexity has been proposed as a mechanism promoting diversity in these systems (Buss and Jackson 1979, Karlson and Jackson 1981). For assemblages of scleractinian corals, there is some debate about the prevalence of intransitive loops (Lang 1973, Connell 1976, Tanner 1992), but broad consensus that competitive outcomes depend on colony state, particularly colony size (e.g., Connell 1976, Lang and Chornesky 1990). Therefore, this paper focuses on the latter phenomenon, examining whether and how size-dependent competition for space can promote coexistence in assemblages of sessile, clonal organisms such as scleractinian corals.

Among scleractinians, eight different kinds of competitive mechanisms have been observed or implicated (Lang and Chornesky 1990), and these can be divided into indirect and direct interactions (Connell 1976). In indirect interactions, the tissues of competitors do not come into direct physical contact. The most prevalent case is overtopping, in which a three-dimensional branching, tabular, or foliaceous coral occupies the space above a colony in the understory. Other growth forms (encrusting and submassive corals) cannot overtop, but they do engage in direct competitive interactions, such as digestion and smothering by overgrowth where tissues of different colonies come into contact (Lang and Chornesky 1990). Direct and indirect competition may both lead to the displacement of one colony by the other, as the loser is digested, smothered, or overtopped. However, direct competition may also result in cessation of growth along the margin of contact between two colonies ("standoffs"), or in "competitive reversals" in which one colony temporarily displaces a portion of another colony, then the pattern is reversed (Connell 1976, Chornesky 1989, Lang and Chornesky 1990).

Although much of the empirical literature on coral competition focuses on competitive encounters between adults, corals also compete by preempting one anothers' larvae. Propagules require space on which to settle, and adults occupy that space. As a result, recruitment decreases as space occupied by adults increases (Hughes 1985, Connell et al. 1997). At the population level, this inhibition of recruitment decreases a species' per capita population growth rate, and thus contributes to competitive-interaction strength (Connolly and Roughgarden 1999). Because both larval preemption and adult-adult interactions occur among scleractinian corals, competitive relationships between species can be quite complex. For instance, colonies of an overtopping species, such as a tabular Acropora, and a digesting understory species, such as a mussid or favid, will preempt one another's larvae. Mussid or favid colonies are likely to be able to digest small, juvenile Acropora (Lang and Chornesky 1990). Conversely, canopy-forming Acropora can overtop mussids and favids (Stimson 1985, Baird and Hughes 2000).

The competitive interactions described above occur between nearby individuals, but the extent to which they influence species coexistence depends upon how important those interactions are at the population level. In particular, the extent to which available space can be monopolized by potential community dominants is limited by the frequency and intensity of mortality events (e.g., disturbances), and by the rate at which recruits colonize unoccupied space (Connell et al. 1997). Models of hierarchical competition suggest that the outcome of competition varies along gradients in mortality and recruitment because competitive ability influences how species respond to such gradients (Connolly and Roughgarden 1998, 1999, Dial and Roughgarden 1998). Thus, it is reasonable to expect that the effects of gradients in mortality and recruitment on community structure will differ between hierarchically structured communities and communities with more complex networks of interactions.

In this paper we extend classical models of competition for space to incorporate key aspects of the sizedependent competitive relationships that characterize scleractinian coral communities. Specifically, we incorporate the preemption of space from larvae by adults, digestion and overgrowth interactions in the understory, and overtopping of understory colonies by canopy-formers. Our objectives are twofold. Firstly, we examine whether and how the kinds of size-dependent relationships that exist in scleractinian coral communities can promote coexistence. Secondly, we examine whether and how size-dependent competition mediates the effects of recruitment and disturbance on coexistence. Analysis of the models uncovers fundamental differences between hierarchical and size-dependent nontransitive competition, and the analytical tractability afforded by the models' relative simplicity allows those differences to be comprehensively characterized. We find that size-dependent competition for space may promote or inhibit coexistence, depending on the extent to which the canopy-former can sustain itself by clonal 
TABLE 1. Summary of competitive effects in the three models.

\begin{tabular}{lll}
\hline \hline Competition model & Indirect effects & Direct effects \\
\hline Standoff & $C$ overtops $J$ and $U$ & standoffs between $J$ and $U$; all populations preempt larvae \\
Reversal & $C$ overtops $J$ and $U$ & $U$ displaces $J$; all populations preempt larvae \\
Hierarchical & $C$ overtops $J$ and $U$ & $C$ and $J$ preempt larvae; $J$ recruits to and overgrows $U$ \\
\hline
\end{tabular}

Note: Key to abbreviations: $C=$ canopy-former, adult stage; $J=$ canopy-former, subcanopy juvenile stage; $U=$ understory population.

propagation. We also find that the qualitative effects of gradients in disturbance and recruitment on the outcome of competition depend fundamentally upon how species compete for space.

\section{Models}

We formulate and analyze three models (Table 1). All include overtopping of understory corals by canopy-formers, but they differ in their characterization of direct competitive encounters in the understory, and of interactions between larvae and adults. In standoff competition (Model I), adults preempt larvae: larvae cannot settle on space occupied by adults, regardless of species. Similarly, direct competition between colonies in the understory is preemptive: colonies cannot competitively displace one another. In reversal competition (Model II), adults also preempt larvae, as in the standoff model. However, in the reversal model the understory species can digest and overgrow colonies of the canopy-forming species before the canopy-formers have matured to canopy height. These standoff and reversal models are formulated to approximate competition between canopy-formers and understory species whose competitive ability in direct encounters is weak or strong, respectively. By contrast, hierarchical competition (Model III) represents a hypothetical interaction that is strictly hierarchical: the canopyformer displaces the understory species, regardless of life stage. By explicitly comparing this model with the standoff and reversal models, we are able to unambiguously characterize the effects of size-dependent shifts in competitive ability on species coexistence.

\section{Single-species demography}

In standoff and reversal competition, the outcome of competition can depend upon the means by which space is colonized (e.g., a digester can overgrow inferior competitors, but its larvae can recruit only to unoccupied space). Therefore, we extend the classical space-colonization framework (e.g., Hastings 1980, Tilman 1994) to differentiate between clonal expansion (colony growth and fragmentation) and larval recruitment in the dynamics of an understory species:

$$
\frac{d U}{d \tau}=b_{U}(1-U) U+g_{U}(1-U) U-m_{U} U .
$$

$U$ is percentage cover (explicit indication of time-dependence, i.e., $U(\tau)$, has been omitted throughout the paper to make the equations more readable); $b_{U}$ is recruitment ability: the proportional rate at which available space is occupied by recruit cover, and $g_{U}$ is clonal growth ability: the proportional rate at which available space is colonized by clonal expansion. Recruitment and clonal expansion exhibit density-dependent, logistic dynamics: both are zero when there is no existing cover to sexually or clonally reproduce $(U=0)$, but both are also zero when there is no free space to colonize $(U=1)$. Partial and total colony mortality is density independent, occurring at rate $m_{U}$ per unit area of cover.

We divide the overtopper population into two stages: a subcanopy juvenile stage, which grows vertically, and a canopy-forming adult stage, which expands horizontally (cf. tabular Acropora growth, Stimson 1985):

$$
\begin{aligned}
\frac{d J}{d \tau} & =b_{C} C F-v_{J} J-m_{J} J-g_{C} C J \\
\frac{d C}{d \tau} & =v_{J} J+g_{C} C(1-C)-m_{C} C \\
F & =1-C-J .
\end{aligned}
$$

$J$ and $C$ represent cover of juvenile (subcanopy) and adult (canopy-forming) colonies, respectively, at time $\tau$. Successive terms in Eq. 2a represent recruitment, maturation to canopy height by vertical growth, density-independent mortality, and overtopping by adults, respectively. Because reproduction is usually initiated after the subcanopy phase (Wallace 1985a, Hall and Hughes 1996), we model the production of potential recruit cover as a function of adult canopy cover, but not juvenile subcanopy cover $\left(b_{C} C\right)$. Recruitment is proportional to free space, $F$ (corals do not recruit to living coral, and rarely recruit underneath canopy cover; Wallace 1985b, Baird and Hughes 2000). Overtopping by tabular corals is typically associated with substantially elevated mortality rates (Stimson 1985, Baird and Hughes 2000), so we model overtopping as a source of juvenile mortality (final term in Eq. 2a). Subcanopy juvenile cover matures to canopy height at rate $v_{J}$ (first term in Eq. 2b), and adult colonies expand horizontally into available space at rate $g_{C}$ (second term in Eq. 2b). Mortality from sources other than competition (e.g., predation, disturbance) occurs at densityindependent rates $m_{J}$ and $m_{C}$ for juveniles and adults, respectively. 


\section{Model 1: Standoff competition}

Standoffs are a likely outcome of direct encounters in the understory between vertically growing juvenile canopy-formers (e.g., staghorn or tabular Acropora, foliaceous Turbinaria) and understory species that lack strong digestive ability (e.g., Montipora, Porites, encrusting Acropora). We model such interactions as follows:

$$
\begin{aligned}
\frac{d U}{d t} & =\left(\rho \beta_{U}+\gamma_{U}\right) U F-\left(\delta \mu_{U}+\gamma_{C} C\right) U \\
\frac{d J}{d t} & =\rho \beta_{C} C F-\left(1+\delta \mu_{J}+\gamma_{C} C\right) J \\
\frac{d C}{d t} & =J+\gamma_{C} C(1-C)-\delta \mu_{C} C \\
F & =1-U-J-C .
\end{aligned}
$$

To facilitate analysis, Eq. 3 has been nondimensionalized using a characteristic rate of $v_{J}\left(t=v_{J} \tau\right.$; Gurney and Nisbet 1998). Thus, $\beta, \gamma$, and $\mu$ represent rates of recruitment, growth, and mortality, respectively, relative to the rate at which juvenile canopy-formers reach canopy height. Recruitment can occur only to space that is neither occupied by juvenile or understory cover, nor shaded by canopy. Standoff competition implies that growth of the understory species likewise occurs only to unoccupied space (i.e., the understory species cannot displace juvenile subcanopy cover, nor does it grow into space shaded by adult canopy-formers). Mortality of the understory population and subcanopy juveniles occurs as adult canopy-formers overtop them.

To facilitate the characterization of coexistence along gradients in disturbance and recruitment, we introduce the parameters $\delta$ and $\rho$, respectively (cf. Hastings 1980, Sebens and Thorne 1985, Dial and Roughgarden 1998, Abrams 2001); $\delta$ represents environmental effects on mortality rate, and $\rho$ represents environmental effects on recruitment ability, and thus can be considered a "recruitment facilitation" parameter. (Conversely, its reciprocal, $1 / \rho$, can be considered a measure of "recruitment limitation," defined here as the degree to which environmental conditions inhibit the rate at which propagules colonize free space.) Note that $\delta$ and $\rho$ modify the population-level averages of mortality rate and recruitment ability. Thus, characterizing patterns of coexistence along these gradients indicates how community structure would be expected to change as the population as a whole was subject to changes in disturbance rates or levels of recruitment limitation. This approach is appropriate for exploring patterns in community structure associated with longterm or geographical variation in average disturbance rates or levels of recruitment limitation (e.g., crossshelf or alongshore on the Great Barrier Reef, Puotinen et al. 1997, Hughes et al. 1999), but not necessarily patterns in community structure along small-scale gradients in disturbance or recruitment within local com- munities (e.g., along a wave exposure gradient within a single reef).

\section{Model II: Reversal competition}

A stronger from of nontransitivity is competitive reversal, in which the understory population displaces juvenile canopy-formers by overgrowth or digestion, but is still susceptible to overtopping by adult canopyformers. Such interactions characterize competition between canopy-formers and encrusting and sub-massive species with strong digestive ability (e.g., species in the families Faviidae and Mussidae). We model such interactions as follows:

$$
\begin{aligned}
\frac{d U}{d t} & =\rho \beta_{U} U F+\gamma_{U} U(F+J)-\left(\delta \mu_{U}+\gamma_{C} C\right) U \\
\frac{d J}{d t} & =\rho \beta_{C} C F-\left(1+\delta \mu_{J}+\gamma_{C} C+\gamma_{U} U\right) J \\
\frac{d C}{d t} & =J+\gamma_{C} C(1-C)-\delta \mu_{C} C \\
F & =1-U-J-C .
\end{aligned}
$$

This differs from the standoff model in that the understory species can displace juvenile subcanopy cover by digestion or overgrowth (second term of Eq. $4 \mathrm{a}$ and final term of Eq. 4b).

Clonal expansion of canopy-formers via fragmentation shares properties with both recruitment and colony growth. Field experiments show that colony fragments can bond to and grow on colonies of species with poor digestive ability (Tanner 1997). This phenomenon suggests that, for the standoff model, space colonization by canopy-formers via fragmentation is best viewed as a component of clonal growth ability rather than recruitment ability. However, it seems unlikely that the fragment of a canopy-former could successfully attach to and grow on a strong digester-the fragment would probably be digested. Thus, in situations better approximated by the reversal model, the establishment of canopy-former fragments may occur principally to unoccupied space, and thus may be more appropriately viewed as a component of recruitment ability than colony growth.

\section{Model III: Hierarchical competition}

To assess the extent to which nontransitive competition promotes or inhibits coexistence, we contrast the above models with a hypothetical system of hierarchical competition. To do this, we formulate a competition model for populations whose single-species demography is identical to those of the understory and canopy-forming populations, but whose interspecific competition is hierarchical: 


$$
\begin{aligned}
\frac{d U}{d t} & =\left(\rho \beta_{U}+\gamma_{U}\right) U F-\left(\delta \mu_{U}+\gamma_{C} C+\rho \beta_{C} C\right) U \\
\frac{d J}{d t} & =\rho \beta_{C} C(1-C-J)-\left(1+\delta \mu_{J}+\gamma_{C} C\right) J \\
\frac{d C}{d t} & =J+\gamma_{C} C(1-C)-\delta \mu_{C} C . \\
F & =1-U-J-C .
\end{aligned}
$$

This model differs from the nontransitive models in that the canopy-former can both recruit to and overgrow space occupied by the understory species. This approach is consistent with classical hierarchical-competition theory, in which propagules of the dominant displace subordinates (e.g., Hastings 1980, Tilman 1994). The model is principally formulated for comparison with the standoff and reversal models, but it can also be considered an approximation of interactions between canopy-forming corals and non-scleractinian organisms to which coral larvae can recruit directly (e.g., coralline algae).

\section{Analysis}

We base our analysis on comparison of species coexistence in the three models. Initially, we conducted an isocline analysis to identify the range of possible outcomes of competition for each of the models. We then addressed our two specific objectives. Firstly, to determine the extent to which standoffs and competitive reversals in the understory facilitate or inhibit coexistence, relative to one another and to hierarchical competition, we contrasted the range of demographic abilities (combinations of growth, recruitment, and mortality parameters) that allow an understory species to coexist with a canopy-former in the three models. Secondly, to determine whether and how the effects of gradients in disturbance and recruitment limitation on species coexistence are altered by nontransitive competition, we contrasted patterns of coexistence along gradients of $\delta$ and $\rho$, respectively, in the three models.

\section{RESUlts}

\section{Isocline analysis}

Isocline analyses indicated that the possible outcomes of competition are qualitatively different for hierarchical competition than for standoff and reversal competition (Appendix A). In the hierarchical model, the understory population has no effect on the canopyformer ( $U$ does not appear in Eqs. 5a or 5b), so the only possible outcomes of competition are exclusion of the understory species or coexistence. For standoff and reversal competition, however, the potential outcomes of competition depend critically upon the relationship between two parameter values: canopy-former growth ability $\left(\gamma_{C}\right)$ and mortality $\left(\mu_{C}\right)$. When $\gamma_{C}$ $>\mu_{C}$, canopy-former persistence is independent of understory abundance, just as for hierarchical competi- tion; therefore, standoff and reversal competition admit only the same two outcomes as hierarchical competition: exclusion of the understory species or coexistence. By contrast, when $\gamma_{C}<\mu_{C}$, exclusion of the canopy-former by the understory species becomes possible. As a result, potential outcomes of competition include exclusion of the understory species by the canopy-former, exclusion of the canopy-former by the understory species, stable coexistence, or a priority effect.

\section{Size-dependent competition and coexistence}

To illustrate how the size-dependent competition affects coexistence, we define two demographic quantities: understory colonization ability and understory longevity. Understory colonization ability is the understory population's intrinsic capacity to colonize space $\left(\beta_{U}+\gamma_{U}\right)$. Understory longevity is the expected length of time that space occupied by the understory species remains occupied $\left(1 / \mu_{U}\right)$. (Because this analysis focuses on differences between life histories, the environmental gradient parameters $\rho$ and $\delta$ are omitted.) We can then examine, for each of the three models, the range of colonization abilities and longevities for which an understory species coexists with a canopyformer, and thereby determine whether coexistence is facilitated or inhibited as competition shifts from hierarchical to standoff to reversal.

For standoff and hierarchical competition, space colonization by sexual recruitment and clonal growth are functionally equivalent: $\beta_{U}$ and $\gamma_{U}$ appear as a sum (Eqs. 3 and 5). Thus, colonization ability and longevity completely describe the understory species' demography. By contrast, under reversal competition, recruitment can occur only to free space, but the understory species can directly displace subcanopy juveniles by clonal propagation. Thus, reversal competition can lie anywhere along a continuum of standoff-like competition (where space colonization is dominated by recruitment, $\beta_{U} \gg \gamma_{U}$ ), and an extreme reversal (where space colonization is dominated by clonal growth, $\gamma_{U}$ $\gg \beta_{U}$ ). By illustrating the standoff and extreme reversal cases, we span the range of outcomes possible under reversal competition.

As with the isocline analysis, results differ qualitatively depending upon the relative magnitudes of canopy growth ability $\left(\gamma_{C}\right)$ and adult canopy-former mortality $\left(\mu_{C}\right)$. When $\gamma_{C}>\mu_{C}$, nontransitivity promotes coexistence. That is, as competition shifts from hierarchical (Fig. 1A), to standoff (Fig. 1B), to reversal (Fig. 1C), a broader range of combinations of understory colonization ability and longevity allow the understory species to persist in the presence of a canopyformer, while the canopy-former persists in the presence of the understory species, regardless of the understory species' demographic abilities. By contrast, when $\gamma_{C}<\mu_{C}$, nontransitivity inhibits coexistence. Coexistence under hierarchical competition still requires the understory species to exceed a threshold combi- 




\begin{tabular}{|c|c|}
\hline s higl & 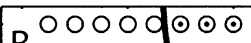 \\
\hline & B $\circ \circ \circ \circ \circ \odot \odot \odot$ \\
\hline$\vec{m}$ &  \\
\hline &  \\
\hline
\end{tabular}

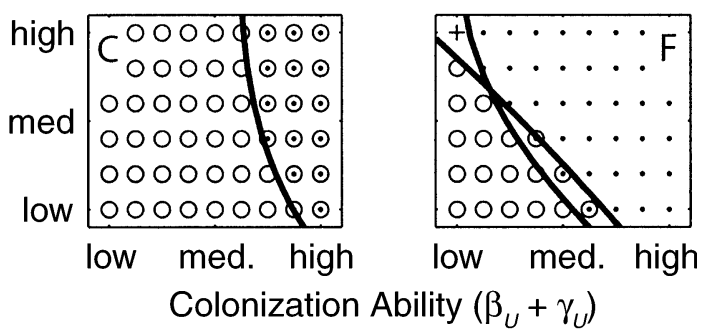

FIG. 1. Effect of nontransitivity on the conditions for coexistence as functions of the understory species' space-colonization ability and longevity for (A, D) hierarchical, (B, E) standoff, and $(\mathrm{C}, \mathrm{F})$ reversal competition. Outcomes of competition are shown as: understory species excluded $(\bigcirc)$, stable coexistence $(\odot)$, canopy-former excluded $(\cdot)$, and priority effect $(+)$. The solid lines demarcate boundaries in parameter space between each of these outcomes of competition. The left-hand column of panels (A, B, C) illustrates outcomes when the canopy-former's clonal growth ability exceeds its mortality rate $\left(\gamma_{C}>\mu_{C}\right)$. The right-hand column of panels (D, E, F) illustrates outcomes when the canopy-former's mortality rate exceeds its clonal growth ability $\left(\mu_{C}>\gamma_{C}\right)$.

nation of colonization ability and longevity (Fig. 1D). By contrast, the standoff and reversal models exhibit a "coexistence bandwidth" (sensu Armstrong 1976), bounded to the left and below by threshold combinations of colonization ability and longevity below which the understory species is excluded, and above and to the right by threshold combinations of those quantities above which the canopy-former is excluded (Fig. 1E and F). Comparison of the standoff and reversal models indicates that further increases in the degree of sizedependent nontransitivity have a qualitatively similar effect: the coexistence bandwidth becomes narrower, and coexistence becomes less likely, as competition shifts from standoff (Fig. 1E) to reversal (Fig. 1F). This outcome occurs because the upper threshold to the coexistence region is reduced more sharply than the lower threshold as one moves from standoff to reversal competition. In other words, increasing nontransitivity inhibits the canopy-former to a greater extent than it benefits the understory species.
Associated with this transition to a coexistence bandwidth is the potential for invasibility thresholds to cross. This outcome indicates the appearance of a region of priority effect (Fig. 1E and F). Moving upwards and leftwards in Fig. 1, understory colonization ability decreases and understory longevity increases. Thus, the location of the priority-effect region above and to the left of the coexistence region indicates that coexistence tends to be inhibited by understory life histories that are characterized by low spatial turnover. Mathematical analysis further supports this inference (Appendix B).

\section{Coexistence along environmental gradients}

In the previous section, we varied the understory species' demographic parameters and held the canopyformer's constant. This approach indicated how coexistence conditions were modified as the species' relative demographic abilities changed. In this section, we hold the competitors' relative demographic abilities constant, and examine how coexistence conditions change with variation in environmental conditions (the disturbance parameter $\delta$ and the recruitment facilitation parameter $\rho$ ). This approach reveals whether and how the nature of competition mediates the effect of environmental gradients on species coexistence.

Competitive outcomes are plotted for cases in which the understory species has a recruitment advantage $\left(\beta_{U}\right.$ $>\beta_{C}$, Fig. 2 A-C), a growth advantage $\left(\gamma_{U}>\gamma_{C}\right.$, Fig. $2 \mathrm{D}-\mathrm{F})$, and a longevity advantage $\left(\mu_{U}<\mu_{C}\right.$, Fig. $2 \mathrm{G}-$ I). Under hierarchical competition, coexistence occurs at intermediate-disturbance levels-as disturbance increases, the outcome of competition shifts from canopy-former only, to coexistence, to the understory species only (illustrated by line 1 in Fig. 2A). For recruitment facilitation, there is a threshold disturbance value below which the understory species cannot persist, regardless of the level of recruitment facilitation (e.g., along line 2 in Fig. 2D). Above this threshold, there is a pattern of coexistence at intermediate levels of recruitment facilitation, with the outcome of competition shifting from the understory species only, to coexistence, to the canopy-former only as recruitment facilitation increases (e.g., along line 3 in Fig. 2G).

Standoff and reversal competition produce patterns of coexistence that differ markedly from those produced by hierarchical competition. As under hierarchical competition, there is a threshold disturbance level below which the canopy-former excludes the understory species, regardless of the degree of recruitment facilitation. However, there is also a threshold disturbance level above which the understory species excludes the canopy-former, regardless of the degree of recruitment limitation (e.g., line 4 in Fig. $2 \mathrm{H}$ ). Thus, coexistence is confined to a band of intermediate-disturbance intensities. Moreover, within this band, increasing recruitment facilitation does not necessarily produce a shift from dominance by the understory species, to coexistence, to dominance by the canopy-for- 
78


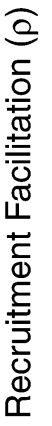

10W

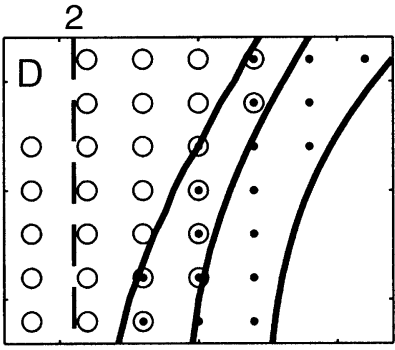

3

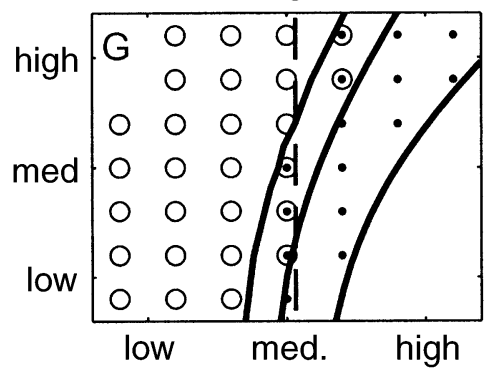

5



4



6

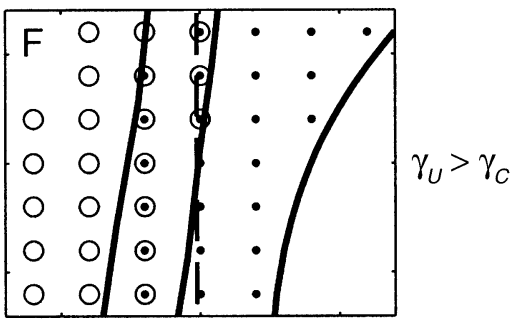

FIG. 2. Outcomes of competition along gradients in disturbance and recruitment facilitation. From left to right in each panel, the regions shown indicate canopy-former only $(\bigcirc)$, coexistence $(\odot)$, understory species only $(\cdot)$, and neither species persists (blank region). The first column of panels (A, D, G) shows hierarchical competition, the second (B, E, H) shows standoff competition, and the third $(\mathrm{C}, \mathrm{F}, \mathrm{I})$ shows reversal competition. The top row $(\mathrm{A}, \mathrm{B}, \mathrm{C})$ shows the case for which the understory species has a recruitment advantage $\left(\beta_{U}>\beta_{C}\right)$; the middle row (D, E, F) shows the case for which the understory species has a growth advantage $\left(\gamma_{U}>\gamma_{C}\right)$; the bottom row $(\mathrm{G}, \mathrm{H}, \mathrm{I})$ shows the case for which the understory species has a longevity advantage $\left(\mu_{U}<\mu_{C}\right)$. The horizontal and vertical dashed lines indicate how the outcome of competition varies along gradients in disturbance or recruitment. Line 1 shows the change in the outcome of hierarchical competition as disturbance increases. Lines 2 and 3 show the possible changes in the outcome of hierarchical competition as recruitment increases. Lines 4-8 indicate the additional effects of recruitment gradients possible under standoff and reversal competition.

mer, as it does under hierarchical competition. Rather, the outcome of competition may involve only part of this sequence, such as from coexistence to dominance by the canopy-former (e.g., along line 5 in Fig. 2E), or from dominance by the understory species to coexistence (e.g., along line 6 in Fig. 2F). Moreover, which species is favored as recruitment increases may change. In particular, when the understory species has a recruitment advantage $\left(\beta_{U}>\beta_{C}\right)$, increasing recruitment facilitation promotes persistence of the understory species when disturbance is low, but it promotes persistence of the canopy-former when disturbance is high (e.g., along lines 7 and 8, respectively, in Fig. $2 B)$. In other words, the qualitative effect of recruit- ment facilitation on coexistence depends upon the level of disturbance-in marked contrast to hierarchical competition.

\section{Discussion}

Size-dependent competition for space between clonal animals promotes coexistence when clonal growth is adequate for persistence of a canopy-former, but not when clonal growth is inadequate. This context dependence differs qualitatively from previous theory of stage-dependent shifts in competitive abilities, which has emphasized the potential for ontogenetic shifts in competitive ability to promote coexistence via tradeoffs in competitive ability at different life stages (Lo- 
reau and Ebenhoh 1994, McCann 1998). We suspect that this difference is related to two features of the models analyzed here: the dual modes of reproduction associated with these organisms (sexual and clonal propagation), and the potential for trade-offs between displacement ability (digestion and overtopping) and resource exploitation ability (space preemption), as well as the more commonly modeled trade-offs in relative competitive ability with size.

Colony growth and fragmentation allows species to circumvent the increased vulnerability to competition that is often associated with early post-settlement life, a developmental option that unitary organisms lack. When clonal growth ability exceeds mortality among adult canopy-formers $\left(\gamma_{C}>\mu_{C}\right)$, the canopy-former is immune to competitive exclusion. Thus, increasing nontransitivity shifts the competitive balance from juvenile canopy-formers toward the understory species, and facilitates persistence of the understory species without excluding the canopy-former. This result arises from the possibility for indeterminate growth of adult canopy-former clones. However, because there are structural constraints to colony size in overtoppers (they will fall over if the canopy becomes too large), this scenario is likely to be most relevant for canopyformers that form multiple attachments to the substrate. For instance, staghorn Acropora, canopy-formers in reef-flat and lagoonal habitats, often have high fragment production and survivorship (Smith and Hughes 1999). Similarly, in turbid waters the expanding edges of foliaceous Turbinaria appear to form secondary attachments to the substrate (S. R. Connolly, personal observation). By contrast, when clonal growth alone is inadequate for persistence $\left(\gamma_{C}<\mu_{C}\right)$, size-dependent shifts in competitive ability inhibit coexistence. Under these conditions, exclusion of the canopy-former is possible, so the coexistence region shifts from being bounded only on one side, by exclusion of the understory species, to a coexistence bandwidth that narrows as competition shifts from standoff to reversal. This scenario is likely to better approximate competition involving canopy-formers that have clear structural constraints to colony size, and that (a) do not fragment, or (b) have fragment survivorship and growth that is too low to compensate for colony mortality (e.g., tabular Acropora, Smith and Hughes 1999).

Our models also differ from existing competition theory by including both direct interference (digestion and overtopping) and indirect exploitation (space preemption). Previous models of ontogenetic shifts in competitive ability have focused on exploitative competition (Loreau and Ebenhoh 1994, McCann 1998). In these cases, the dominant competitor is the species capable of persisting when the limiting resource is at its lowest level (Tilman 1982, Huisman and Weissing 1994). In such systems, stage-dependent shifts promote coexistence by means of a trade-off between exploitation ability in one stage vs. another stage. By contrast, in space competition the competitive dominant is typically defined as the species whose individuals are capable of directly displacing individuals of the other species, while the species capable of most efficiently exploiting the limiting resource (space) is the one with the colonization and/or longevity advantage (e.g., Hastings 1980). Incorporation of direct displacement makes possible a trade-off between interference and exploitation ability, even in the absence of niche partitioning, much like the classical competition-colonization trade-off (Yu and Wilson 2001). This trade-off is not possible in the purely exploitative systems characterized in previous work. In this study, differences between models in the effects of this trade-off on coexistence overwhelm any effects of stage-dependent shifts in resource exploitation ability, and thus drive the predicted relationships between size-dependent competition and coexistence.

Our results also show that the qualitative effects of disturbance and recruitment depend upon the nature of competition. Behavior of the hierarchical model presented here is broadly consistent with that of an hierarchical model for intertidal barnacles (Dial and Roughgarden 1998), despite differences in model detail. However, disturbance and recruitment affect coexistence under standoff and reversal competition very differently than under hierarchical competition. For standoffs and reversals, there is an upper disturbance threshold to coexistence, above which the understory species excludes the canopy-former. This upper threshold produces a stronger intermediate-disturbance pattern than under hierarchical competition, with threshold disturbance rates below and above which coexistence is impossible, regardless of the degree of recruitment limitation. By contrast, the corresponding intermediate-recruitment phenomenon appears to be sensitive to the nature of competition, as it is largely eliminated by standoffs and reversals. This is not to say that the degree of recruitment limitation has no effect on coexistence. In particular, the coexistence region can broaden as recruitment is increasingly facilitated (e.g., Fig. 2B and C), in contrast to the conventional view that recruitment limitation promotes coexistence by preventing the monopolization of resources. Numerical investigations indicate that this expansion of the coexistence region with increasing recruitment holds whenever the understory species has a recruitment advantage. It can also occur in the absence of a recruitment advantage, if the understory species' growth or longevity advantage is sufficiently large.

Finally, one unexpected finding is worth highlighting: the tendency for low spatial turnover in the understory to inhibit coexistence under standoff and reversal competition (Fig. 1E and F and Appendix B). The counterintuitive nature of this result is best illustrated by example. For an understory population in the absence of a canopy-former population (Eq. 1), the 
percentage of unoccupied space at equilibrium is $\hat{F}=$ $\mu_{U} /\left(\gamma_{U}+\beta_{U}\right)$. Now, consider two different understory populations. Both have the same $\hat{F}$, but one has high turnover (i.e., high mortality, balanced by high recruitment and growth ability), while the other has low turnover. Under standoff competition, the competitive effect of the understory population on the canopy-former is strictly via preemption of larval recruitment by adults. The amount of space that is not preempted $(\hat{F})$ is the same for both understory populations, so one might expect the two populations to be equally invasible by a canopy-former. Previous theory for purely symmetric, preemptive competition supports this intuition: invasibility depends on $\hat{F}$ of the resident, not the demographic details of how that $\hat{F}$ is maintained (Iwasa and Roughgarden 1986). However, this prediction changes, and intuition is violated, for standoff and reversal competition: the population with low turnover is more difficult to invade. This result is especially counterintuitive for reversal competition: increased colony growth ability of the understory population should lead to an increased rate of encounters with, and digestion of, juvenile canopy-formers. Thus, one might expect that a digester with high turnover (i.e., higher clonal growth, balanced by higher mortality) would be more difficult to invade. However, we find precisely the opposite.

\section{Conclusions}

Coral-reef ecology has lacked a theoretical framework for assessing how complex competitive interactions influence species coexistence on coral reefs, relative to environmental processes that mediate the effects of competition at the population level, such as disturbance and recruitment limitation (Knowlton and Jackson 2001). Our results show that, even under recruitment-limited conditions (i.e., space is not exhausted), and in the presence of high mortality, conditions for coexistence differ substantially and often qualitatively under hierarchical vs. size-dependent, nontransitive competition. Moreover, the nature of competition can qualitatively alter how gradients in recruitment limitation and disturbance influence coexistence. There is growing recognition that ontogenetic shifts in competitive relationships can exert substantial influence on patterns of species coexistence (Werner and Gilliam 1984, Loreau and Ebenhoh 1994, Werner 1994, McCann 1998), and such shifts are known to be widespread among clonal invertebrates (Jackson and Buss 1975, Buss and Jackson 1979, Sebens 1982, Lang and Chornesky 1990). These observations, and the results presented here, call for a renewed emphasis on the effects of size-dependent competition, and other mechanisms that cause nontransitive competitive networks in benthic communities.

\section{ACKNOWLEDGMENTS}

We thank K. Anthony, A. Baird, J. Caley, M. Hixon, T. Hughes, J. Huisman, R. Karlson, and an anonymous reviewer for thoughtful and constructive comments on this manuscript. This research was supported by grants from the Australian Research Council and James Cook University, awarded to S. R. Connolly, and a postdoctoral fellowship from the Japanese Society for the Promotion of Science, awarded to S. Muko. This is contribution number 70 of the Centre for Coral Reef Biodiversity (CCRB), and contribution number 196 of the Coral Ecology Group at James Cook University.

\section{Literature Cited}

Abrams, P. A. 2001. The effect of density-independent mortality on the coexistence of exploitative competitors for renewing resources. American Naturalist 158:459-470.

Armstrong, R. A. 1976. Fugitive species: experiments with fungi and some theoretical considerations. Ecology 57: 953-963.

Baird, A. H., and T. P. Hughes. 2000. Competitive dominance by tabular corals: an experimental analysis of recruitment and survival of understorey assemblages. Journal of Experimental Marine Biology and Ecology 251:117-132.

Buss, L. W. 1980. Competitive intransitivity and size-frequency distributions of interacting populations. Proceedings of the National Academy of Sciences (USA) 77:53555359.

Buss, L. W., and J. B. C. Jackson. 1979. Competitive networks: nontransitive competitive relationships in cryptic coral reef environments. American Naturalist 113:223-234.

Chesson, P. L., and R. R. Warner. 1981. Environmental variability promotes coexistence in lottery competitive systems. American Naturalist 117:923-943.

Chornesky, E. A. 1989. Repeated reversals during spatial competition between corals. Ecology 70:843-855.

Connell, J. H. 1961. The influence of interspecific competition and other factors on natural populations of the barnacle Chthamalus stellatus. Ecology 42:710-723.

Connell, J. H. 1976. Competitive interactions and the species diversity of corals. Pages 51-58 in G. O. Mackie, editor. Coelenterate ecology and biology. Plenum Press, New York, New York, USA.

Connell, J. H. 1978. Diversity in tropical rain forests and coral reefs. Science 199:1302-1310.

Connell, J. H., T. P. Hughes, and C. C. Wallace. 1997. A 30year study of coral abundance, recruitment, and disturbance at several scales in space and time. Ecological Monographs 67:461-488.

Connolly, S. R., and J. Roughgarden. 1998. A latitudinal gradient in intertidal community structure: evidence for an oceanographically-based synthesis of marine community theory. American Naturalist 151:311-326.

Connolly, S. R., and J. Roughgarden. 1999. Theory of marine communities: competition, predation, and recruitment-dependent interaction strength. Ecological Monographs 69: 277-296.

Dial, R., and J. Roughgarden. 1998. Theory of marine communities: the intermediate disturbance hypothesis. Ecology 79:1412-1424.

Dungan, M. L. 1985. Competition and the morphology, ecology, and evolution of acorn barnacles: an experimental test. Paleobiology 11:165-173.

Gurney, W. S. C., and R. M. Nisbet. 1998. Ecological dynamics. Oxford University Press, Oxford, UK.

Hall, V. R., and T. P. Hughes. 1996. Reproductive strategies of modular organisms: comparative studies of reef-building corals. Ecology 77:950-963.

Hastings, A. 1980. Disturbance, coexistence, history and competition for space. Theoretical Population Biology 18: 363-373.

Hubbell, S. P. 2001. The unified neutral theory of biodiversity and biogeography. Princeton University Press, Princeton, New Jersey, USA. 
Hughes, T. P. 1985. Life histories and population dynamics of early successional corals. Pages 101-105 in C. Gabrie, J. L. Taffart, and B. Salvat, editors. Proceedings of the Fifth International Coral Reef Congress, Tahiti. Volume 4. Attenne Museum-EPHE, Moorea, French Polynesia.

Hughes, T., A. Baird, E. Dinsdale, N. Moltschaniwskij, M. Pratchett, J. Tanner, and B. Willis. 1999. Patterns of recruitment and abundance of corals along the Great Barrier Reef. Nature 397:59-63.

Huisman, J., and F. J. Weissing. 1994. Light-limited growth and competition for light in well-mixed aquatic environments: an elementary model. Ecology 75:507-520.

Hutchinson, G. E. 1961. The paradox of the plankton. American Naturalist 95:137-145.

Iwasa, Y., and J. Roughgarden. 1986. Interspecific competition among metapopulations with space-limited subpopulations. Theoretical Population Biology 30:194-214.

Jackson, J. B. C. 1979. Overgrowth competition between encrusting cheilostome ectoprocts in a Jamaican cryptic reef environment. Journal of Animal Ecology 48:805-823.

Jackson, J. B. C., and L. Buss. 1975. Allelopathy and spatial competition among coral reef invertebrates. Proceedings of the National Academy of Science (USA) 72:5160-5163.

Karlson, R. H. 1980. Alternative competitive strategies in a periodically disturbed habitat. Bulletin of Marine Science 30:894-900.

Karlson, R. H. 1983. Disturbance and monopolization of a spatial resource by Zoanthus sociatus (Coelenterata, Anthozoa). Bulletin of Marine Science 33:118-131.

Karlson, R. H., and J. B. Jackson. 1981. Competitive networks and community structure: a simulation study. Ecology 62:670-678.

Keddy, P. A., and B. Shipley, 1989. Competitive hierarchies in herbaceous plant communities. Oikos 54:234-241.

Knowlton, N., and J. B. C. Jackson. 2001. The ecology of coral reefs.Pages 395-422 in M. D. Bertness, S. D. Gaines, and M. E. Hay, editors. Marine community ecology. Sinauer Associates, Sunderland, Massachusetts, USA.

Lang, J. 1973. Interspecific aggression by scleractinian corals. 2. Why the race is not only to the swift. Bulletin of Marine Science 23:260-279.

Lang, J. C., and E. A. Chornesky. 1990. Competition between scleractinians reef corals - a review of mechanisms and effects. Pages 209-252 in Z. Dubinsky, editor. Coral reefs. Ecosystems of the World, Volume 25. Elsevier, Amsterdam, The Netherlands.

Loreau, M., and W. Ebenhoh. 1994. Competitive exclusion and coexistence of species with complex life cycles. Theoretical Population Biology 46:58-77.

McCann, K. 1998. Density-dependent coexistence in fish communities. Ecology 78:2957-2967.

Muko, S., and Y. Iwasa. 2000. Species coexistence by permanent spatial heterogeneity in a lottery model. Journal of Theoretical Biology 57:273-284.

Munday, P. L., G. P. Jones, and M. J. Caley. 2001. Interspecific competition and coexistence in a guild of coral-dwelling fishes. Ecology 82:2177-2189.

Paine, R. T. 1966. Food web complexity and species diversity. American Naturalist 100:65-75.

Puotinen, M. L., T. J. Done, and W. C. Skelly. 1997. An atlas of tropical cyclones in the Great Barrier Reef region, 1969-
1997. Technical Report Number 19. CRC Reef Research Centre, Townsville, Queensland, Australia.

Sale, P. 1977. Maintenance of high diversity in coral reef fish communities. American Naturalist 111:337-359.

Sebens, K. 1982. Competition for space: growth rate, reproductive output, and escape in size. American Naturalist 120:189-197.

Sebens, K. P. 1986. Spatial relationships among encrusting marine organisms in the New England subtidal zone. Ecological Monographs 56:73-96.

Sebens, K., and B. Thorne. 1985. Coexistence of clones, clonal diversity, and the effects of disturbance. Pages 347399 in J. B. C. Jackson, L. Buss, and R. Cook, editors. Population biology and evolution of clonal organisms. Yale University Press, New Haven, Connecticut, USA.

Smith, L. D., and T. P. Hughes. 1999. An experimental assessment of survival, reattachment and fecundity of coral fragments. Journal of Experimental Marine Biology and Ecology 235:147-164.

Stimson, J. P. 1985. The effect of shading by the table coral Acropora hyacinthus on understory corals. Ecology 66:4053.

Stone, L. 1995. Biodiversity and habitat destruction: a comparative study of model forest and coral reef ecosystems. Proceedings of the Royal Society of London B 261:381388.

Tanner, J. 1992. Experimental analysis of digestive hierarchies in coral assemblages. Pages 569-574 in R. H. Tanner, editor. Proceedings of the Seventh International Coral Reef Symposium, Guam. University of Guam Press, Mangilao, Guam.

Tanner, J. 1997. Interspecific competition reduces fitness in scleractinian corals. Journal of Experimental Marine Biology and Ecology 214:12-34.

Tanner, J. E., T. P. Hughes, and J. H. Connell. 1994. Species coexistence, keystone species, and succession: a sensitivity analysis. Ecology 75:2204-2219.

Tanner, J. E., T. P. Hughes, and J. H. Connell. 1996. The role of history in community dynamics: a modelling approach. Ecology 77:108-117.

Tilman, D. 1982. Resource competition and community structure. Princeton University Press, Princeton, New Jersey, USA.

Tilman, D. 1994. Competition and biodiversity in spatially structured habitats. Ecology 75:2-16.

Wallace, C. C. $1985 a$. Reproduction, recruitment and fragmentation in nine sympatric species of the coral genus Acropora. Marine Biology 88:217-233.

Wallace, C. C. 1985 b. Seasonal peaks and annual fluctuations in recruitment of juvenile scleractinian corals. Marine Ecology Progress Series 21:289-298.

Werner, E. 1994. Ontogenetic scaling of competitive relations: size-dependent effects and responses in two anuran larvae. Ecology 75:197-213.

Werner, E. E., and J. F. Gilliam. 1984. The ontogenetic niche and species interactions in size-structured populations. Annual Review of Ecology and Systematics 15:393-425.

Yu, D., and H. Wilson. 2001. The competition-colonization trade-off is dead; long live the competition-colonization trade-off. American Naturalist 158:49-63.

\section{APPENDIX A}

Results of the invasibility analysis are available in ESA's Electronic Data Archive: Ecological Archives E084-078-A1.

\section{APPENDIX B}

Mathematical analysis of the effects of understory turnover rate on coexistence is available in ESA's Electronic Data Archive: Ecological Archives E084-078-A2. 